\title{
Cochlear Implantation in Isolated Large Vestibular Aqueduct Syndrome: Report of Three Cases and Literature Review
}

\author{
Rabindra Pradhananga ${ }^{1,2}$ Kiran Natarajan ${ }^{3}$ AmarNath Devarasetty ${ }^{3}$ Mohan Kameswaran ${ }^{2}$ \\ ${ }^{1}$ Department of ENT-Head and Neck Surgery, Tribhuvan University \\ Teaching Hospital, Kathmandu, Nepal \\ 2 Department of Implantation Otology, Madras ENT Research \\ Foundation, Chennai, Tamil Nadu, India \\ ${ }^{3}$ Department of Otorhinolaryngology, Madras ENT Research \\ Foundation, Chennai, Tamil Nadu, India

\begin{abstract}
Address for correspondence Rabindra Pradhananga, MS, Fellowship in Implantation Otology; Department of ENT-Head and Neck Surgery, Tribhuvan University Teaching Hospital, Kathmandu, Nepal, Kathamandu +977, Nepal (e-mail: rabindrabp@yahoo.com; rabindra9034@gmail.com).
\end{abstract}

Int Arch Otorhinolaryngol 2015;19:359-363.

\begin{abstract}
Keywords

- large vestibular aqueduct

- cochlear implantation

- CSF gusher

Introduction Large vestibular aqueduct syndrome (LVAS) is characterized by the enlargement of the vestibular aqueduct associated with sensorineural hearing loss. It is the most common radiographically detectable inner ear anomaly in congenital hearing loss. LVAS may occur as an isolated anomaly or in association with other inner ear malformations.

Objective To report three cases of isolated LVAS with a focus on preoperative assessment, surgical issues, and short-term postoperative follow-up with preliminary auditory habilitation outcomes.

Resumed Report One girl and two boys with LVAS were assessed and cochlear implantation was performed for each. Various ways of intraoperative management of cerebrospinal fluid gusher and postoperative care and outcomes are reported.

Conclusion Cochlear implantation in the deaf children with LVAS is feasible and effective.
\end{abstract}

\section{Introduction}

The vestibular aqueduct (VA) is a tiny, bony canal in the otic capsule that originates on the medial wall of the vestibule and extends toward the cerebellar face of the petrous pyramid at the posterior cranial fossa. It contains the endolymphatic duct connecting the endolymphatic sac and the vestibule. It has an average diameter of 0.6 to $1.5 \mathrm{~mm}$ at its midpoint between the common crus and its opening at the posterior cranial fossa. ${ }^{1,2}$ The VA derives from a diverticulum formed in the wall of the otocyst during the fifth week. The aqueduct begins as a short, broad pouch but gradually elongates and thins until it achieves its characteristic J shape of adulthood. ${ }^{3}$ A premature arrest in development produces an abnormally short and broad VA.
When this anatomical anomaly of large VA is associated with hearing loss, it is referred as large vestibular aqueduct syndrome (LVAS). LVAS is usually bilateral. Valvassori and Clemis were the first to describe and name LVAS after finding the association between a large VA and sensorineural hearing loss (SNHL) in 50 cases. $^{1}$

Cochlear implantations were performed in 99 congenital prelingual deaf children of less than 6 years in the first 5 months of 2014 in our institute. Among them, isolated LVAS was detected in three. The aim of the study was to report these three cases of isolated LVAS focusing mainly on preoperative assessment, surgical issues, and short-term postoperative follow-up with preliminary auditory habilitation outcomes measured in terms of Categories of Auditory received

August 24, 2014

accepted

October 11, 2014

published online

November 14, 2014
DOI http://dx.doi.org/

10.1055/s-0034-1395791. ISSN 1809-9777.
Copyright $\odot 2015$ by Thieme Publicações License terms Ltda, Rio de Janeiro, Brazil 
Table 1 Category of Auditory Performance

\begin{tabular}{|l|l|}
\hline Category & Criteria \\
\hline 7 & Uses telephone with known speaker \\
\hline 6 & Understands conversation without lip-reading \\
\hline 5 & $\begin{array}{l}\text { Understands common phrases without } \\
\text { lip-reading }\end{array}$ \\
\hline 4 & $\begin{array}{l}\text { Discriminates speech sounds without } \\
\text { lip-reading }\end{array}$ \\
\hline 3 & Identifies environmental sounds \\
\hline 2 & Responds to speech sounds \\
\hline 1 & Aware of environment sounds \\
\hline 0 & Not aware of environmental sounds \\
\hline
\end{tabular}

Performance (CAP; - Table 1 ) and Speech Intelligibility Rating (SIR; - Table 2) scores.

\section{Literature Review}

Enlargement of the VA is diagnosed radiographically, when its anteroposterior diameter exceeds $1.5 \mathrm{~mm}$ on computed tomography (CT) scan of temporal bone, measured midway between its aperture and crus communes. ${ }^{1,4}$ In many cases, LVA accompanies malformation of the cochlea and/or SCCS (semi-circular canals). It also may be the sole radiographically detectable abnormality of the inner ear in a child with hearing loss (isolated LVAS).

More commonly, LVAS is associated with nonsyndromic deafness. But it can also be associated with syndromic hearing loss as in Pendred syndrome, branchio-oto-renal syndrome, and CHARGE syndrome (coloboma of the eye, heart defects, atresia of the nasal choanae, retardation of growth and/or development, genital and/or urinary abnormalities, and ear abnormalities and deafness). It has been postulated that LVAS is inherited as an autosomal recessive trait. ${ }^{5}$

Cochlear implantation effectively increases both auditory perception and speech and language development in children with LVAS. ${ }^{6}$ Most of the literature reported cerebrospinal fluid (CSF) gusher or leak as a common problem encountered during and after cochlear implantation. ${ }^{6}$ Miyamoto et al published results of a retrospective case-control study on outcomes of cochlear implantation in 23 patients with LVAS and 46 control patients and concluded that cochlear implan-

Table 2 Speech Intelligibility Rating

\begin{tabular}{|l|l|}
\hline Category & Criteria \\
\hline 5 & Intelligible to all listeners \\
\hline 4 & $\begin{array}{l}\text { Intelligible to a listener who has } \\
\text { little experience of a deaf person's speech }\end{array}$ \\
\hline 3 & $\begin{array}{l}\text { Intelligible to a listener who } \\
\text { concentrates and lip-reads }\end{array}$ \\
\hline 2 & Intelligible speech is developing in single words \\
\hline 1 & Unintelligible \\
\hline
\end{tabular}

tation was beneficial for the treatment of hearing loss in LVA as well as in the control patients. ${ }^{7}$ Chen et al compared 62 infants with LVAS for development of auditory skills after cochlear implant and found results similar to those of infants with a normal inner ear. ${ }^{8}$

\section{Case Reports}

\section{Case 1}

A 25-month-old boy presented to the Implant Outpatient Department with a history of trouble hearing, first noticed by the parents at the age of 8 months, with delay in speech and language development. The child was born at full term by cesarean section with a birth weight $1.9 \mathrm{~kg}$. There was no history of maternal illnesses including TORCH [toxoplasmosis, other (syphilis, varicella-zoster, parvovirus B19), rubella, cytomegalovirus, and herpes infection], Rh-incompatibility, neonatal jaundice, or meningitis. His other developmental milestones were within normal limits. There was no history of visual disturbances, hypothyroidism, syncopal spells, or urinary disturbances. General examination showed normal IQ with no neurologic dysfunction. Ear, nose, and throat (ENT) examination showed normal external ear with normal and healthy-looking tympanic membrane. There was no response to tuning fork tests in either ear. Other ENT examination was within normal limits.

The patient underwent detailed audiological workup. Behavioral audiometry showed profound SNHL in both ears. Tympanogram showed A-type curve in both ears. Brainstem evoked response audiometry (BERA) showed absent wave $\mathrm{V}$ on both sides at $90-\mathrm{dB} \mathrm{nHL}$ (normal Hearing Level) at the rate of $19.3 / \mathrm{s}$ using click and tone burst $(500-\mathrm{Hz})$ stimuli. No distortion product otoacoustic emission was detected, which suggested outer hair cell dysfunction. High-resolution computed tomography (HRCT) of the temporal bone showed evidence of LVA with a diameter of $2.8 \mathrm{~mm}$ on both sides (-Fig. 1). He was also assessed by a child psychologist and speech pathologist. The patient and his parents were introduced to other implantees at the habilitation center run by Madras ENT Research Foundation (MERF) with regard to maintenance of the implant and auditory verbal habilitation.

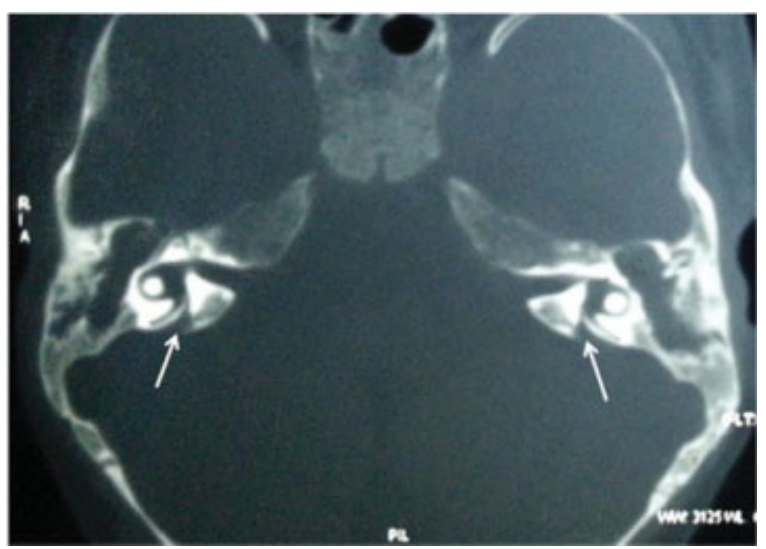

Fig. 1 Computed tomography scan of case 1 showing large vestibular aqueduct. 
He was vaccinated with Quadri Meningo (Meningococcal Polysaccharide: group A, C, Y, and W135 - Bio-Med private Ltd, Ghaziabad, India) and Sii Hib Pro (Haemophilus Type B conjugate - Serum Institute of India Ltd, Pune, India) vaccine.

The patient's right ear was operated on because he was right-handed and had similar inner ear anatomy in both sides. The round window (RW) membrane was exposed via transmastoid facial recess approach. The pulsatile RW membrane was distinct. The incudostapedial (I-S) joint was dislocated, and immediately a pulsatile stapes sign was obvious. On making an incision on the RW membrane, a pulsatile CSF gusher started. Then the head end of the table was raised and intravenous $20 \%$ mannitol drip ( $1.5 \mathrm{~g} / \mathrm{kg}$ body weight over 20 minutes) was started. The gusher was significantly reduced within 10 minutes and the electrode array of the implant inserted via the RW. Intraoperative parental antibiotics were given. Complete insertion of an electrode array up to the mark was achieved. Temporalis muscle fascia was used to seal the fenestrum tightly around the electrode array. Fibrin glue was also used to achieve a watertight seal intraoperatively. The intraoperative telemetry showed satisfactory impedance and neural response in all the electrodes.

Postoperatively one more dose of $20 \%$ mannitol drip ( $1.5 \mathrm{~g} / \mathrm{kg}$ body weight over 20 minutes) was given on the same evening. He was kept with the prophylactic intravenous antibiotics for 3 days. Oral acetazolamide, twice daily, was also used for 3 days.

During follow-up, he did not have any vestibular symptoms or sign of CSF otorhinorrhea. He had no further problem before or after the implant was turned on. After 3 months of auditory habilitation, his CAP and SIR scores were 3 and 2, respectively, which were 2 and 1 before start of habilitation.

\section{Case 2}

A boy 5 years and 9 months old born from consanguineous parents presented to our Implant Outpatient Department with a history of hearing problems noticed by the parents at the age of around 12 months, when the child had not developed speech and language. Antenatal, natal, and postnatal history was normal, as well as family history. His other developmental milestones were within normal limits. He was also assessed by the ENT consultant, audiologist, psychologist, and speech therapist. The entire audiological package

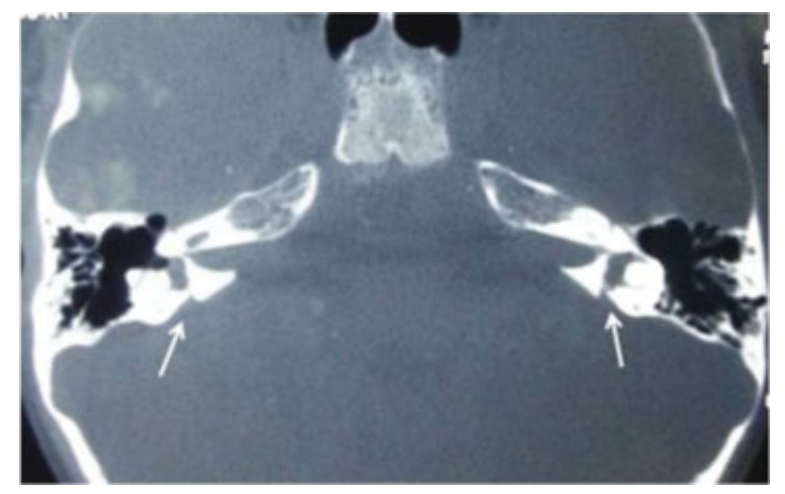

Fig. 2 Computed tomography scan of case 2 showing large vestibular aqueduct. test was performed as in case 1 and the reports were similar, confirming cochlear hearing loss. Temporal bone CT scan showed normal outer, middle, and inner ear structures except an increase in diameter $(2.1 \mathrm{~mm}$ ) of the VA (-Fig. 2). His right ear was operated on. After cortical mastoidectomy and posterior tympanotomy, the RW niche was exposed. In this case also a pulsatile stapes sign was obvious after disarticulation of I-S joint. Due to the anterior position of the facial nerve, the RW membrane could not be exposed and cochleostomy was performed. Immediately after cochleostomy, a pulsatile CSF leak started. It was successfully managed intraoperatively as in case 1 except with use of tissue glue. Further management was continued as in case 1 . After 3 months of auditory habilitation, this patient's CAP and SIR scores were 3 and 1, respectively, which were 1 and 1 before start of habilitation.

\section{Case 3}

A girl 4 years and 8 months old was referred from Deaf Screening camp organized in a remote area of Tamil Nadu, India. According to her parents, she did not respond to sound since early childhood and not developed speech and language even after use of hearing aids. She was admitted to a deaf school in the village. She had an uneventful pre- and postnatal period and a birth weight of $3.5 \mathrm{~kg}$. The parents had a history of consanguineous marriage. She was also assessed preoperatively, as in case 1 , and the reports were similar, confirming cochlear hearing loss. Temporal bone CT scan evidenced LVA $(1.9 \mathrm{~mm}$ ) on both sides (-Fig. 3). She also received her implant in the right ear. In this case also a pulsatile RW could clearly be noticed after exposing the RW via standard transmastoid facial recess approach, but the pulsatile stapes sign was not obvious even after dislocating the I-S joint. An incision was made on the RW membrane. Immediately after the puncture, CSF started leaking in a pulsatile manner. After elevating the head end of the operating table, the gusher decreased significantly. Within a few minutes, the CSF gusher was well controlled without a mannitol drip. Then electrode array was inserted via the RM. The last two pairs of electrodes could not be inserted as a resistance could be felt at the end. Temporalis muscle fascia was used to seal the fenestrum

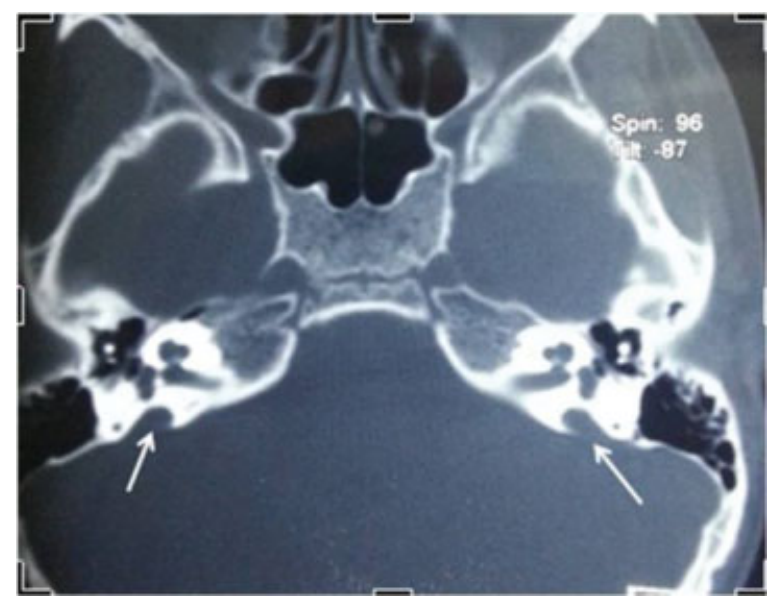

Fig. 3 Computed tomography scan of case 3 showing bilateral large vestibular aqueduct. 
around the electrode array tightly. Further leak was not present. Postoperatively, she did not develop any vestibular dysfunction or CSF otorhinorrhea. After 3 months of auditory habilitation, her CAP and SIR scores were improved from 2 to 4 and from 1 to 2 , respectively.

\section{Discussion}

LVA is the most common inner ear malformation that is detectable via radiologic imaging. ${ }^{1}$ It can occur as an isolated anomaly or in association with other inner ear malformation like Mondini dysplasia. Valvassori and Clemis found the size of VA ranged from 1.5 to $8 \mathrm{~mm}$ in the anteroposterior diameter; however, the diameter of the aqueduct of all the present cases ranged from 1.9 to $2.8 \mathrm{~mm}$.

The exact incidence of LVA in prelingual deaf children is still underestimated; we found nearly $3 \%$. Emmett reported up to $12 \%$ incidence of LVAS, but not in prelingual deaf cases ${ }^{9}$; however, Fahy et al found a $4 \%$ incidence. ${ }^{10}$ Children with LVA are usually born with normal (4\%) or mildly impaired hearing that gradually deteriorates through childhood into adolescence and early adulthood. Hearing levels vary, although at least $39 \%$ eventually develop profound sensory hearing loss. ${ }^{11}$ There is also a tendency to suffer sudden decrements of hearing, particularly after mild head trauma. Govaerts et al found that $90 \%$ of the cases with LVA had mixed hearing loss and a steady decrease of hearing at an average rate of $4 \mathrm{~dB} / \mathrm{y}{ }^{12}$ Because we are reporting cases with prelingual deafness before cochlear implantation, the variability of hearing status in LVA was out of the realm of the study.

During the surgery, all the steps of the cochlear implantation remain similar to normal cases. RW insertion is preferred over cochleostomy in our center nowadays. Out of three cases, two had RW pulsation clearly visible after exposing the RW membrane. The I-S joint was disarticulated to demonstrate clear pulsatile stapes sign. This pulsatile stapes sign described earlier by the authors was discernible in two cases. ${ }^{13}$ The authors believe that the presence of pulsatile stapes predicts the severity of CSF gusher. Immediately after incision on RW or cochleostomy, pulsatile CSF gusher was noted in all three cases. Although CSF gusher is a well-known surgical risk in all patients with LVA, no gusher was experienced by Harker et al in cochlear implantation in five LVAS patients, ${ }^{14}$ and Fahy et al experienced gusher in two of four cases. ${ }^{10}$

Various techniques to control the CSF leak have been described and can be utilized according to the severity of the leak. We used some techniques like elevation of head end of table, mannitol, tight sealing the insertion site, tissue glue, and postoperative oral acetazolamide to control CSF gusher/ leak. To reduce the CSF gusher during surgery, we used mannitol drip in two cases immediately after the incision to the RW membrane or cochleostomy. It was well controlled in all three cases after sealing the site of insertion with temporalis muscle fascia graft around the electrode array after the insertion. CSF leak in LVA may be controlled without any extra technique except tightly packing the cochleostomy or insertion site. Tissue glue was used only in a severe gusher to get a watertight seal.
Intraoperative management of CSF leak may require complete packing of the middle ear space in addition to the cochleostomy. A lumbar drain is rarely necessary. In inner ear malformation, a gusher at surgery is directly related to the intracerebral pressure (ICP). The mainstay of hyperosmolar treatment is to reduce the ICP at the time of the surgery. Loundon et al suggested osmotherapy as an effective means for control of leakage during cochleostomy in LVA, which could be effective in more severe malformations. ${ }^{15}$

Acetazolamide has become a standard treatment for CSF leaks associated with intracranial hypertension. Though its role is not established in cochlear implantation, we routinely use acetazolamide in all CSF gushers as it reduces intracranial pressure and helps control further leakage.

Vaccination and antibiotic prophylaxis against meningitis were similar in all the cases. In all three cases, MedEl SONATA (MedEl Elektromedizinische Gerate $\mathrm{GmbH}$, Austria) + standard implant was used.

On the day of operation, two patients had an episode of vomiting, but all were discharged within 72 hours of surgery. There was no problem noticed in subsequent follow-up. The implant was switched on 3 weeks postoperatively, and the postoperative progress of these children in listening skills was similar to other implantees. The outcomes on hearing and speech development are being followed, but preliminary assessment with CAP and SIR score at 3 months showed no difference from other normal implantees.

\section{Final Comments}

The results of the present cases and the review of the literature suggest that implantation in LVAS can be performed without intra- and postoperative complications. The outcomes on hearing and speech development are being followed, but preliminary assessment showed no difference compared with implantees with normal cochlea at 3 months' follow-up. Therefore, cochlear implantation in deaf children with LVAS is feasible and effective.

\section{References}

1 Valvassori GE, Clemis JD. The large vestibular aqueduct syndrome. Laryngoscope 1978;88(5):723-728

2 Becker TS, Vignaud J, Sultan A, Lachman M. The vestibular aqueduct in congenital deafness: evaluation by the axial projection. Radiology 1983;149(3):741-744

3 Bagger-Sjoback D, Jansson B, Friberg U, Rask-Andersen H. Threedimensional anatomy of the human endolymphatic sac. Arch Otolaryngol Head Neck Surg 1990;116(3):345-349

4 Dahlen RT, Harnsberger HR, Gray SD, et al. Overlapping thin-section fast spin-echo MR of the large vestibular aqueduct syndrome. AJNR Am J Neuroradiol 1997;18(1): 67-75

5 Abe S, Usami S, Shinkawa H. Three familial cases of hearing loss associated with enlargement of the vestibular aqueduct. Ann Otol Rhinol Laryngol 1997;106(12):1063-1069

6 Aschendorff A, Marangos N, Laszig R. Large vestibular aqueduct syndrome and its implication for cochlear implant surgery. Am J Otol 1997;18(6, Suppl):S57 
7 Miyamoto RT, Bichey BG, Wynne MK, Kirk KI. Cochlear implantation with large vestibular aqueduct syndrome. Laryngoscope 2002;112(7 Pt 1):1178-1182

8 Chen X, Liu B, Liu S, et al. The development of auditory skills in infants with isolated Large Vestibular Aqueduct Syndrome after cochlear implantation. Int J Pediatr Otorhinolaryngol 2011;75(7):943-947

9 Emmett JR. The large vestibular aqueduct syndrome. Am J Otol 1985;6(5):387-415

10 Fahy CP, Carney AS, Nikolopoulos TP, Ludman CN, Gibbin KP. Cochlear implantation in children with large vestibular aqueduct syndrome and a review of the syndrome. Int J Pediatr Otorhinolaryngol 2001;59(3):207-215

11 Jackler RK, De La Cruz A. The large vestibular aqueduct syndrome. Laryngoscope 1989;99(12):1238-1242, discussion 1242-1243
12 Govaerts PJ, Casselman J, Daemers K, De Ceulaer G, Somers T, Offeciers FE. Audiological findings in large vestibular aqueduct syndrome. Int J Pediatr Otorhinolaryngol 1999;51(3):157-164

13 Natarajan K, Raghunandhan S, Senthilvadivu SM, Kameswaran M. The pulsatile stapes: an unusual sign in large vestibular aqueduct syndrome. Indian Journal of Otolaryngology \& Head \& Neck Surgery 2010;62(Suppl 1):16-18

14 Harker LA, Vanderheiden S, Veazey D, Gentile N, McCleary E. Multichannel cochlear implantation in children with large vestibular aqueduct syndrome. Ann Otol Rhinol Laryngol Suppl 1999; 177:39-43

15 Loundon N, Leboulanger N, Maillet J, et al. Cochlear implant and inner ear malformation. Proposal for an hyperosmolar therapy at surgery. Int J Pediatr Otorhinolaryngol 2008;72(4):541-547 